\title{
Disruption of an amino acid transporter LHT1 leads to growth inhibition and low yields in rice
}

\author{
Xiaohu Wang ${ }^{1 \dagger}$, Guangzhe Yang ${ }^{1+}$, Mingxing Shi ${ }^{1}$, Dongli Hao², Qiuxing Wei ${ }^{1}$, Zhigang Wang ${ }^{1}$, Shan Fu',
} Yanhua Su${ }^{2}$ and Jixing $\mathrm{Xia}^{1^{*}}$ (D)

\begin{abstract}
Background: Research on plant amino acid transporters was mainly performed in Arabidopsis, while our understanding of them is generally scant in rice. OsLHT1 (Lysine/Histidine transporter) has been previously reported as a histidine transporter in yeast, but its substrate profile and function in planta are unclear. The aims of this study are to analyze the substrate selectivity of OsLHT1 and influence of its disruption on rice growth and fecundity.

Results: Substrate selectivity of OsLHT1 was analyzed in Xenopus oocytes using the two-electrode voltage clamp technique. The results showed that OsLHT1 could transport a broad spectrum of amino acids, including basic, neutral and acidic amino acids, and exhibited a preference for neutral and acidic amino acids. Two oslht1 mutants were generated using CRISPR/Cas9 genome-editing technology, and the loss-of-function of OSLHT1 inhibited rice root and shoot growth, thereby markedly reducing grain yields. QRT-PCR analysis indicated that OsLHT1 was expressed in various rice organs, including root, stem, flag leaf, flag leaf sheath and young panicle. Transient expression in rice protoplast suggested OsLHT1 was localized to the plasma membrane, which is consistent with its function as an amino acid transporter.

Conclusions: Our results indicated that OsLHT1 is an amino acid transporter with wide substrate specificity and with preference for neutral and acidic amino acids, and disruption of OsLHT1 function markedly inhibited rice growth and fecundity.
\end{abstract}

Keywords: Amino acid transporter, OsLHT1, Rice, Electrophysiology

\section{Background}

Nitrogen $(\mathrm{N})$ is one of the major mineral nutrients essential for plants growth and development. Plants absorb $\mathrm{N}$ mainly in the inorganic form (nitrate and ammonium) from soils, while the organic $\mathrm{N}$ such as amino acids could also be absorbed [1, 2]. Upon entering the plants, inorganic $\mathrm{N}$ is converted into amino acids in roots or leaves. The reduced $\mathrm{N}$ is transported mainly in the form of amino acids to developing vegetative or reproductive sink organs, where it is used for growth and development. The uptake and translocation of organic and inorganic $\mathrm{N}$ is mediated by a variety of transporters. Nowadays, the nitrate and

\footnotetext{
* Correspondence: xiajx@gxu.edu.cn

${ }^{+}$Xiaohu Wang and Guangzhe Yang contributed equally to this work.

${ }^{1}$ State Key Laboratory of Conservation and Utilization of Subtropical

Agro-bioresources, College of Life Science and Technology, Guangxi

University, Nanning 530005, China

Full list of author information is available at the end of the article
}

ammonium transporters have been extensively studied in plants [3, 4], while the amino acid transporters are still poorly understood, especially in crop plants.

Physiological studies using isolated membrane vesicles and plant tissues have suggested the presence of multiple amino acid transporters with wide substrate specificity in plants [5-7]. Molecular methods have allowed the cloning of genes responsible for amino acids transport. The first plant amino acid transporters AAP1 and NAT2 were identified by functional complementation of yeast mutants defective in amino acid uptake from Arabidopsis cDNA libraries, both of which exhibited broad substrate specificity $[8,9]$. Thereafter a multitude of amino acid transporters were reported from several plant species $[10,11]$. In recent years, sequencing the full genomes of different plants gave us a chance to grasp the abundance of amino acid transporters in plant genomes.

(c) The Author(s). 2019 Open Access This article is distributed under the terms of the Creative Commons Attribution 4.0 International License (http://creativecommons.org/licenses/by/4.0/), which permits unrestricted use, distribution, and 
At least 67, 85 and 189 genes have been identified to encode putative amino acid transporters in Arabidopsis, rice and soybean, respectively [12-14]. Based on sequence analysis, the amino acid transporters identified so far could be divided into two families: the amino acid/polyamine/choline (APC) family and the amino acid transporter (ATF) family. The ATF family was also referred to as AAAP (amino acid/auxin permease) family, and encompasses at least five subfamilies: AAPs (amino acid permeases), LHTs (lysine/histidine transporters), ProTs (proline transporters), ANTs (aromatics and neutral amino acid transporters) and AUXs (auxin transporters) $[13,15,16]$. These transporters generally differ in substrate specificity/affinity and tissue localization [17].

AtLHT1, the first member identified in LHT family, was initially viewed as a lysine (Lys) and histidine (His) selective transporter [18]. However, subsequent studies with other LHT members revealed that LHTs could transport a broad but distinct spectrum of amino acids [19-21]. LHT genes were expressed in various plant organs, including roots, leaves and flowers. They could mediate not only amino acids uptake from soil, but also translocation and partitioning of amino acids within plants [19-21]. However, it should be noted that the research on LHT transporters was mainly performed in Arabidopsis, while our understanding of LHTs in rice is generally scant. A genome-wide survey identified 6 LHT members in rice [14]. Liu et al. [22] isolated a LHT family member OsHT1 from rice by screening cDNA library, which could restore the growth of yeast mutant defective in His uptake. Due to highest identity with AtLHT1 among the 10 LHT family members in Arabidopsis, OsHT1 is referred to as OsLHT1 hereafter.

Although OsLHT1-mediated His transport was determined in yeast [22], it is not clear at present whether OsLHT1 could transport other amino acids. In this study, the substrate specificity of OsLHT1 was analyzed in Xenopus oocytes, and we found that OsLHT1 was able to transport a broad spectrum of amino acids with preference for neutral and acidic amino acids. Furthermore, to explore the role of OsLHT1 in rice growth and development, two oslht1 mutants were constructed using the CRISPR-Cas9 gene-editing technology. It is found that disruption of OsLHT1 significantly inhibited the rice growth and fecundity. Also, the expression pattern and subcellular localization of OsLHT1 was analyzed. This study will further our understanding of the substrate specificity of OsLHT1 and its roles in rice growth promotion and yield formation.

\section{Results}

Broad substrate specificity of OsLHT1

To analyze the substrate selectivity of OsLHT1, OsLHT1 cRNA was injected into Хепориs oocytes, and two- electrode voltage clamp (TEVC) was employed to record the currents induced by different amino acids [23, 24]. Consistent with its function as a His transporter [22], OsLHT1 could transport His in oocytes (Fig. 1a). Besides His, several other amino acids such as Glu, Asp, Asn, Gly, Pro and Ser, could also be efficiently transported by OsLHT1, while Lys, Gln and Ala were only marginally transported (Fig. 1a, b). In contrast, water-injected control oocytes showed no current responses to external applications of amino acids (Fig. 1a). Unlike AtLHT1 [18], the neutral amino acid Asn was the best substrate for OsLHT1 among all of the amino acids tested (Fig. 1a, b). The kinetics of Asn transport by OsLHT1 was further analyzed in Xenopus oocytes, which is saturable with a $K_{\mathrm{m}}$ value of $583 \pm 51 \mu \mathrm{M}$ (Fig. 1c). These data demonstrate that OsLHT1 is an amino acid transporter with wide substrate specificity and with preferential selectivity for neutral and acidic amino acids.

\section{Knockout of OsLHT1 results in growth inhibition}

To investigate the effect of OsLHT1 disruption on rice growth, the CRISPR-Cas9 approach was employed to generate oslht1 mutants. A CRISPR-Cas9 vector expressing guide RNAs targeting the coding region of OsLHT1 was constructed (Fig. 2a) and transformed into rice (Japonica cv Nipponbare). Two mutants oslht1-1 and oslht1-2 were obtained, and then the genomic DNA of the two mutants was extracted for PCR amplification. Sequencing analysis of PCR products revealed that these two mutants were homozygous and had 1-bp adenine (A) and thymine (T) insertions at the desired target sites, respectively (Fig. 2b). Consequently, mutation of oslht11 led to the conversion of Pro10 in OsLHT1 into Leu and subsequent frameshift; mutation of oslht1-2 led to the conversion of Phe32 in OsLHT1 into Ser and subsequent frameshift. We also tested for potential off-target cleavage by searching the rice genome for sequences with high similarity to the OsLHT1 target sequence using the on-line tool (http://skl.scau.edu.cn/offtarget/). For each target, four of the most likely off-target sites were examined, and no off-target cleavage was detected. Additionally, the transcript level of OsLHT1 was detected, which was significantly reduced in the two oslht 1 lines compared with WT (Additional file 1: Figure S1).

When sowing seeds, the germination rates of oslht1 mutants were lower than that of the wild type (WT) at 2 day after sowing (DAS), while the results are almost the same between the wild type and mutants at 3-5 DAS (Additional file 1: Figure S2). At 5 DAS, the shoot growth of the mutants is the same as the wild-type, but the mutants had much shorter roots than the wild type (Fig. 2c, d). These results suggested that the loss of OsLHT1 function slightly delayed seed germination, but markedly influenced root development. To evaluate the 


\section{a Control}

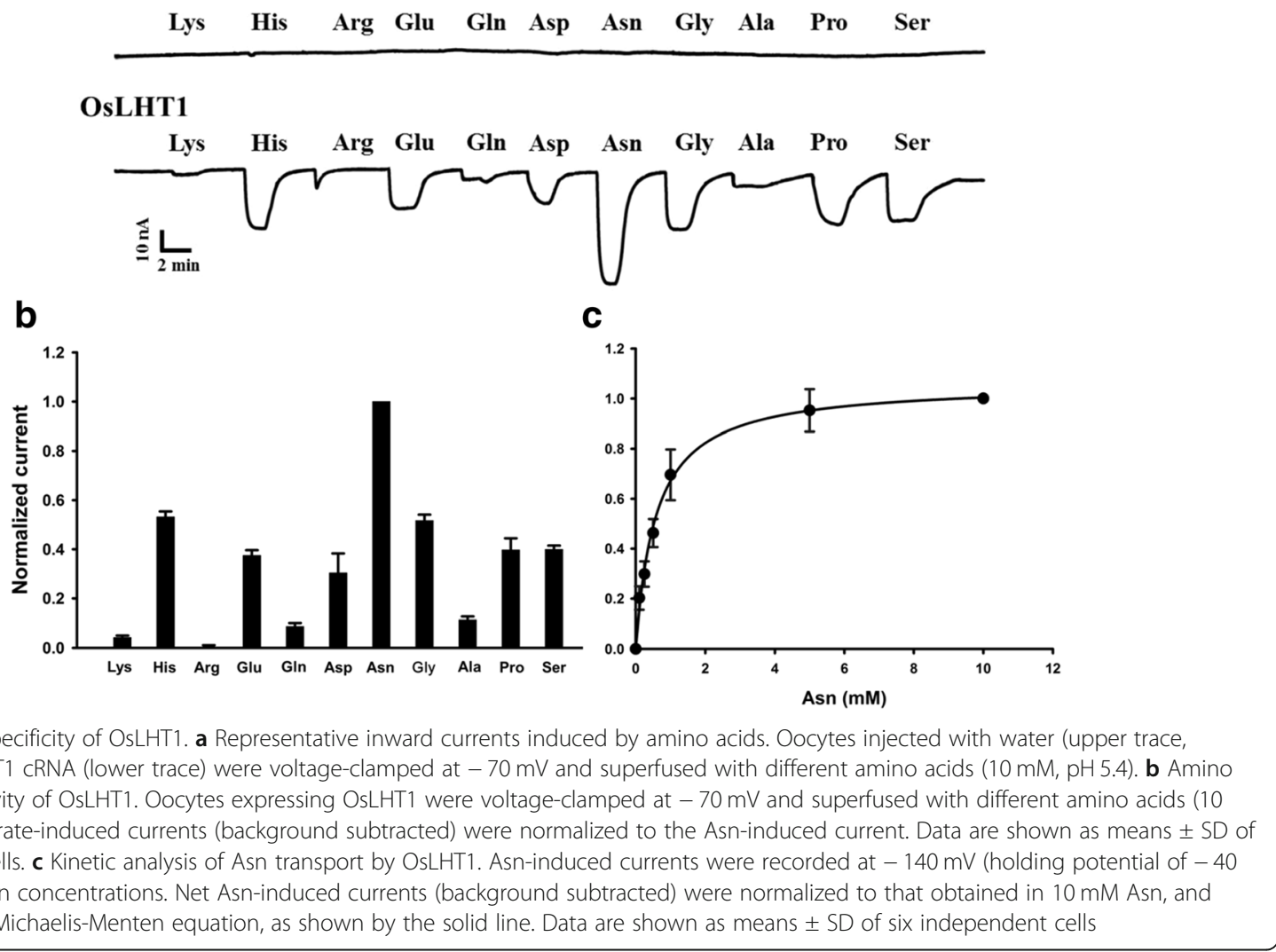

influence of OsLHT1 disruption on the growth over the entire growth period, the wild type and mutants were grown in soil. Compared with the wild type, both mutants oslht1-1 and oslht1-2 exhibited growth inhibition to different extents (Fig. 2e, f). Then the agronomic traits of wild-type and oslht1 plants were further analyzed with respect to plant height, stem length and tiller number. The results showed that, compared with the wild type, the plant height, stem length and tiller number were reduced significantly in the oslht 1 plants (Fig. 2h, i, j). Additionally, during reproductive growth stage, premature senescence was also observed in the oslht1 plants (Fig. 2g). These results indicated that a lack of OsLHT1 function affected plant normal growth and development.

\section{Knockout of OsLHT1 results in low grain yields}

To evaluate the influence of OsLHT1 disruption on the grain yields, we compared the grain yields of oslht $1-1$, oslht $1-2$ and the wild type. We found that the grain yields per plant of oslht 1 were decreased by 67.8 and $42.5 \%$ depending on the mutant line (Fig. 3a, b). Then the yield components, including panicle number per plant, grain number per panicle, seed setting rate and 1000-grain weight, were examined in detail. No significant differences in panicle number per plant were observed between oslht1 and wild-type plants (Fig. 3c). The grain number per panicle of oslht1-1 and oslht1-2 compared with wild-type plants was decreased significantly, by 25 and 14\%, respectively (Fig. $3 \mathrm{~d}$ ). The seed-setting rate was substantially reduced by $42.2 \%$ in oslht $1-1$ and $34.7 \%$ in oslht $1-2$ compared with WT (Fig. 3e), while the 1000-grain weight were reduced between 5.8 and $8.7 \%$ depending on the mutant (Fig. 3f). Additionally, no significant change was observed in grain size and shape in oslht1 plants (Additional file 1: Figure S3). Taken together, these results suggested that disruption of OSLHT1 had a large impact on grain number per panicle and seed setting rate, thus resulting in low yields in oslht1 plants.

Spatial expression and subcellular localization of OsLHT1 To examine the spatial expression of OsLHT1, real-time PCR analysis was carried out to analyze the transcript levels of OSLHT1 in different organs of 14-week-old plants, including root, stem (lower internodes), flag leaf sheath, flag leaf and young panicle. The result showed that OsLHT1 was expressed in all of the rice organs tested, with the lowest expression observed in the lower internodes (Fig. 4a). Further, a more detailed expression of OsLHT1 at different developmental stages was 


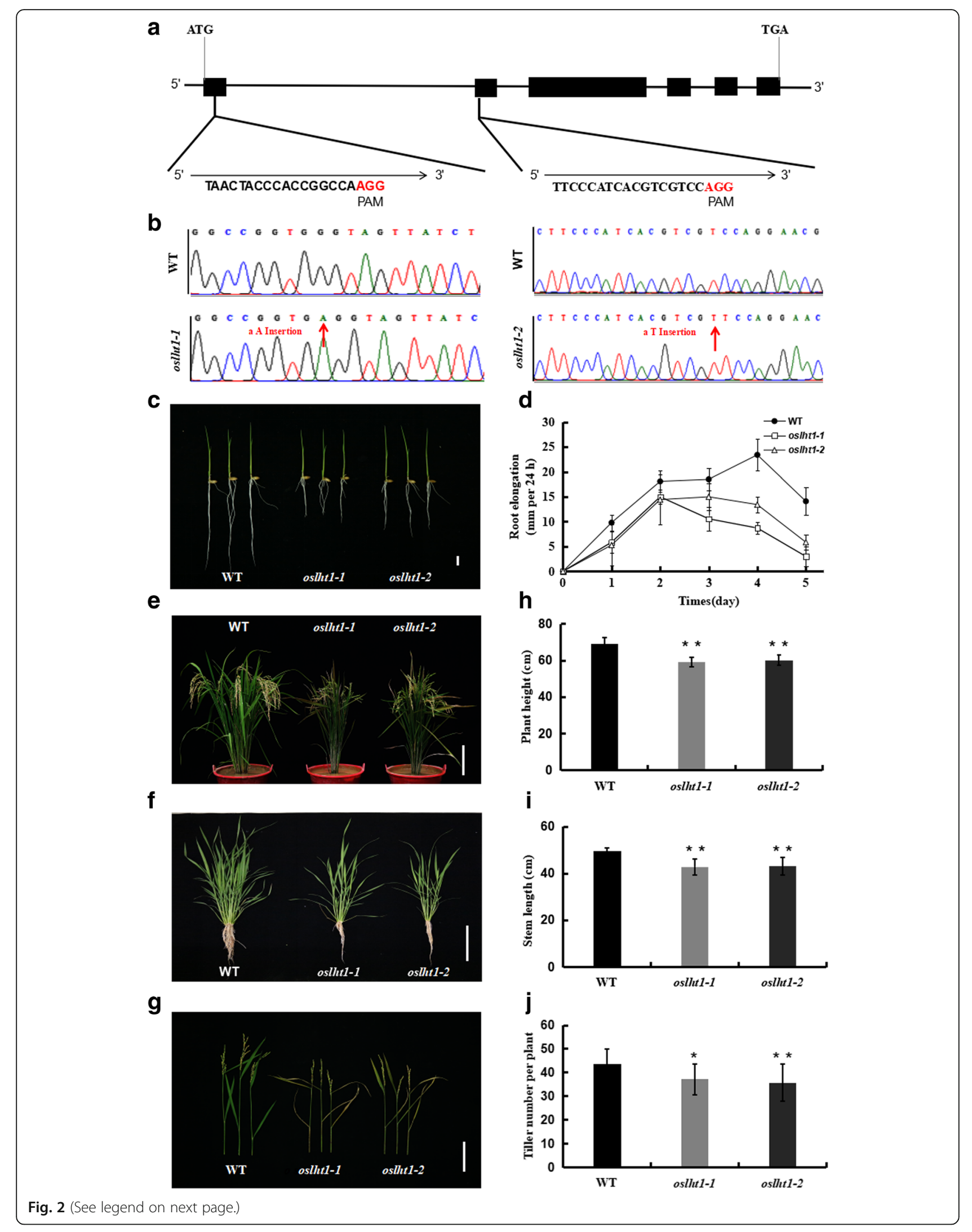


(See figure on previous page.)

Fig. 2 Targeted mutagenesis of OsLHT1 by CRISPR/Cas9 led to rice growth inhibition. a Gene structure of OsLHT1 and the two targeted sites. Black boxes indicate exons. Red letters indicate the PAM of the recognition sequence. $\mathbf{b}$ Sequencing chromatography of wild type, mutants os/ht1-1 and os/ht1-2. Red arrows indicate mutation sites. c Phenotype of 5-day-old WT, oslht1-1 and os/ht1-2 grown on $0.5 \mathrm{mM} \mathrm{CaCl} 2$ solution. Bar $=1 \mathrm{~cm}$. $\mathbf{d}$ Time-dependent root elongation. Germinated seedlings were exposed to a $0.5 \mathrm{mM} \mathrm{CaCl} 2$ solution and the root length was measured at different days. Error bars represent \pm SD $(n=10)$. e-f Gross morphological phenotypes of WT, oslht1-1and os/ht1-2 mutants grown in the field. Bar $=30 \mathrm{~cm}$ (e), $20 \mathrm{~cm}(\mathrm{f}) . \mathbf{g}$ Symptoms of early senescence were present in os $/ \mathrm{ht} 1$ plants at reproductive growth stage. Bar $=20 \mathrm{~cm}$. $\mathbf{h}-\mathbf{j}$ Comparison of plant height (h), stem length (i) and tiller number (j) of the wild type (WT) and oslht1 plants at harvest. Values (h to j) are the mean \pm SD $(n=15)$. Asterisks indicate significant differences from the wild type $\left({ }^{*} P<0.05 ;{ }^{* *} P<0.01\right.$ by Student's $t$-test)

obtained from the electronic Fluorescent Pictograph (eFP) Browser [25], indicating that OsLHT1 exhibited high expression in the developing inflorescence and seed (Additional file 1: Figure S4).

To examine the subcellular localization of OsLHT1, the OsLHT1-GFP fusion proteins under the control of the $35 \mathrm{~S}$ promoter was constructed and transiently expressed in rice protoplasts. After 16-20 h culture, the GFP (green fluorescent protein) fluorescence signal was observed. Protoplasts expressing GFP alone typically showed green fluorescence throughout the cytosol (Fig. 4b). In contrast, in cells expressing OsLHT1-GFP, the green fluorescence was confined to the plasma membrane, co-localized with the plasma membrane-localized small GTPase OsRac3 (mCherry-OsRac3) (Fig. 4b) [26]. The result suggested that OsLHT1 was targeted to the plasma membrane. a

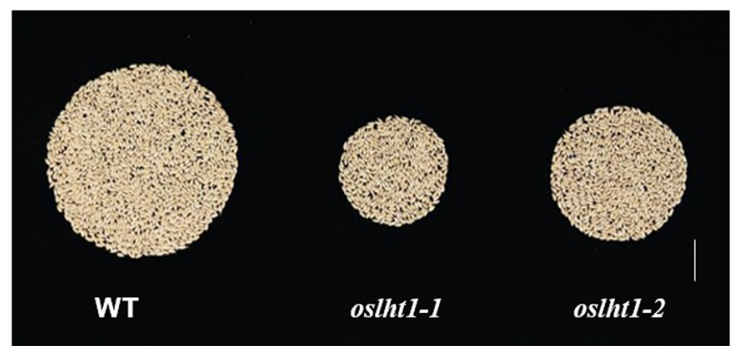

b

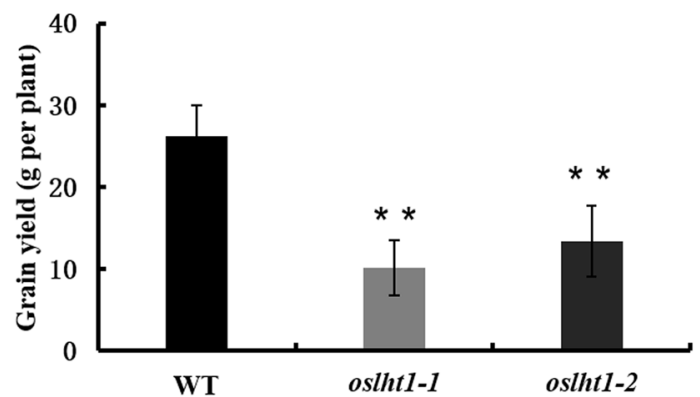

C

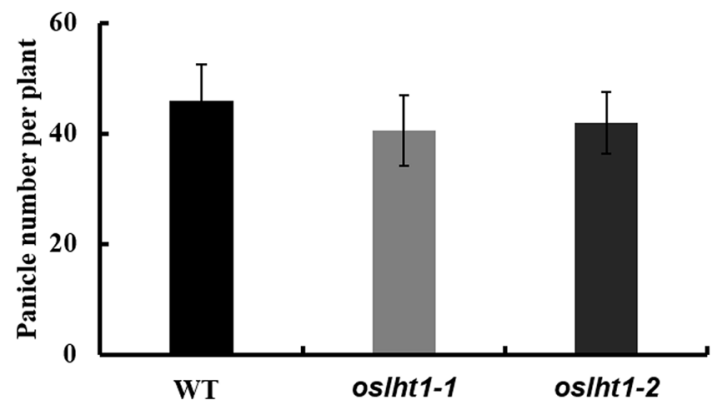

d

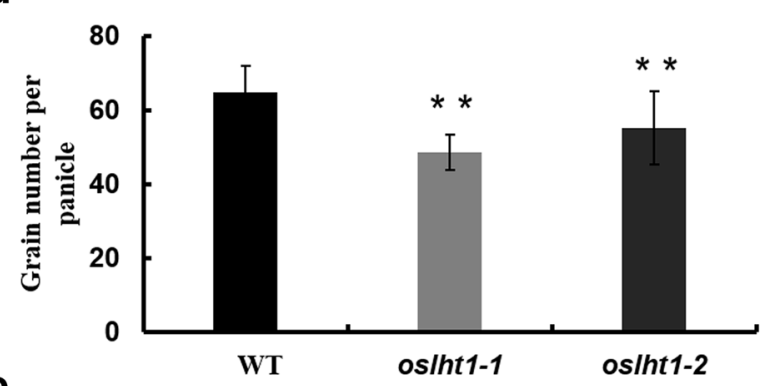

e

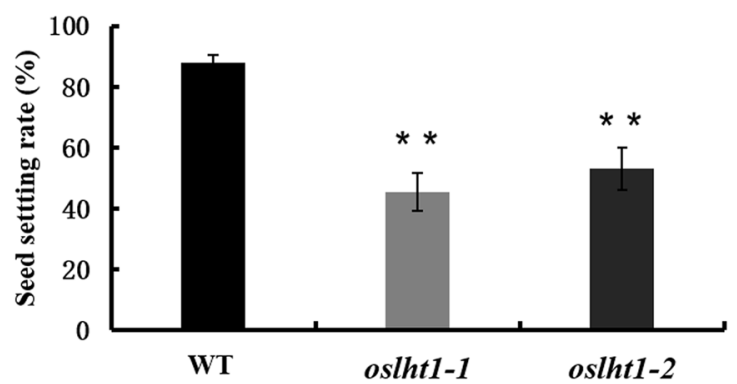

f

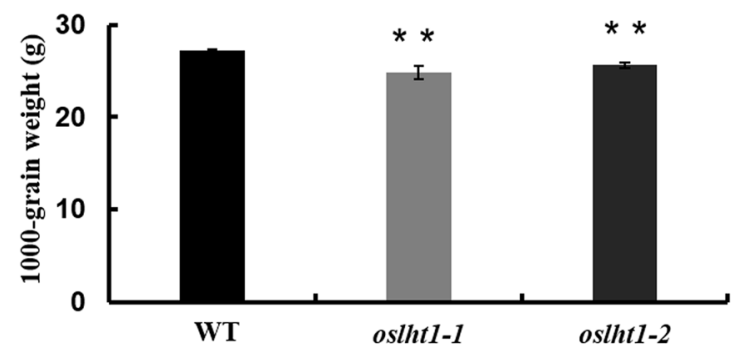

Fig. 3 Loss of OsLHT1 function reduced rice grain yield. a Total grains per plant of WT and os/ht1 plants grown in field. Scale bars, $1 \mathrm{~cm}$. b-f Comparison of grain yield per plant (b), panicle number per plant (c), grain number per panicle (d), seed setting rate (e) and 1000-grain weight (f) of the WT and oslht1 plants. Values (b to $f)$ are the mean \pm SD $(n=15)$. Asterisks indicate significant differences from the wild type $\left({ }^{*} P<0.05\right.$; ${ }^{*} P<0.01$ by Student's $t$-test) 


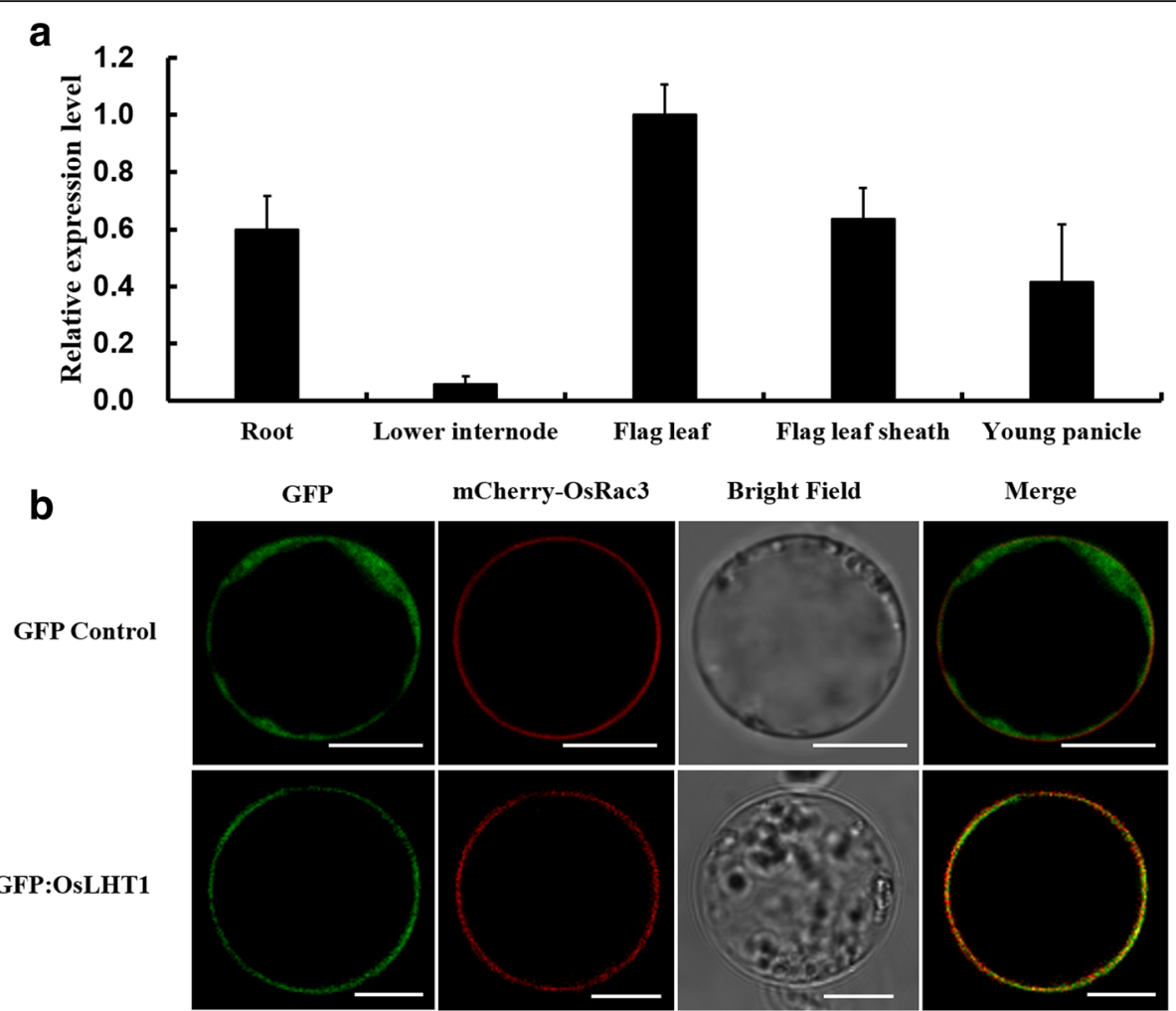

Fig. 4 Organ-specific expression and subcellular localization of OsLHT1. a Expression of OsLHT1 in various organs of the wild-type plants, analyzed by quantitative real-time PCR. The data are shown as the mean \pm SD $(n=3)$. b Subcellular localization of the OsLHT1. GFP:OsLHT1 or GFP was transiently introduced into rice protoplast together with $m$ Cherry-OsRac3 by PEG-mediated transformation. Fluorescence signals from GFP, mCherry-OsRac3, and the merged images are shown. Free GFP was used as a control. Bars, $10 \mu \mathrm{m}$

\section{Phylogenetic analysis of OsLHT1}

OsLHT1 is a member of LHT subfamily that belonged to the amino acid transporter (ATF) family. 6 and 10 LHT genes were identified in rice and Arabidopsis genome, respectively $[14,27]$. We further used the OsLHT1 sequence as a query to search for its homologs in other monocots, and 16,14 and 9 homologs were obtained from maize, sorghum and Brachypodium distachyon, respectively. These proteins, together with LHTs in rice and Arabidopsis, were used for phylogenetic analyses. The resulting phylogenetic tree was mainly divided into two clades, in which OsLHT1 to OsLHT4 belong to one clade, OsLHT5 and OsLHT6 constitute another clade (Fig. 5a). Among the six OsLHTs, OsLHT1 has highest homology to OsLHT2 with identity $76 \%$. The identity of OsLHT1 with its closest homolog in maize (NP 001141364, 90\%), sorghum (XP_021320860, 92\%) and Brachypodium distachyon (XP_003573326, 91\%) are much higher with that in Arabidopsis (AtLHT2, 78\%). Furthermore, the conserved motifs of the six OsLHTs and the closest homologs of OsLHT1 were predicted using the MEME motif search tool. With the exception of OsLHT5 and OsLHT6, the other LHTs have same motifs (Fig. 5b).

\section{Discussion}

AtLHT1 is the first member identified in the LHT subfamily belonging to the amino acid transporter (ATF) family [18]. Uptake measurements in yeast suggested that AtLHT1 transported Lys and His most efficiently among the 13 different amino acids tested, and the $K_{\mathrm{m}}$ values for Lys $(175 \mu \mathrm{M})$ and His $(400 \mu \mathrm{M})$ were much lower than that for the third most-active substrate, Leu $(11 \mathrm{mM})$, so it was regarded as a Lys and His selective transporter [18]. However, studies on the transport properties of other members of LHT family suggested that LHTs were able to transport a wide spectrum of amino acids, including neutral, basic and acidic amino acids, and exhibited preferential selectivity for neutral and acidic amino acids with high affinity [20, 21, 29]. For example, uptake analysis in yeast revealed that AtLHT2 transported aspartate and proline with high affinity $\left(K_{\mathrm{m}}=72,13 \mu \mathrm{M}\right.$, respectively), and competition experiments showed that other neutral and acidic amino acids, but not basic amino acids, act as efficient competitors for proline and aspartate uptake [20]. Furthermore, Perchlik et al. [21] studied AtLHT6 function in planta and found that knockout of AtLHT6 significantly reduced the uptake of acidic and some neutral amino 
a

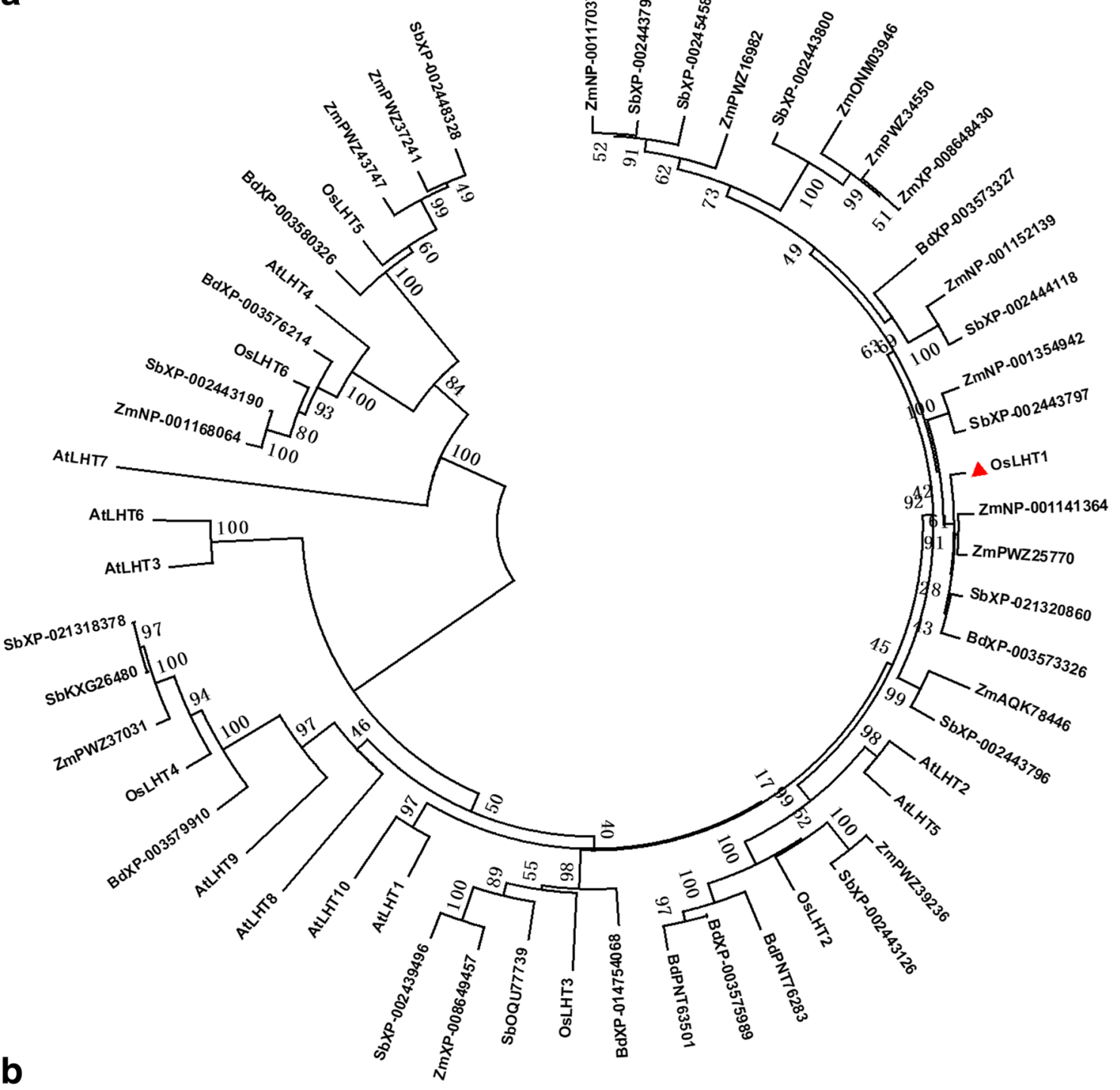

OsLHT1

OsLHT2

OsLHT3

ZmNP 001141364

SbXP 021320860

BdXP 003573326

AtLHT2

OsLHT4

OsLHT6

OsLHT5
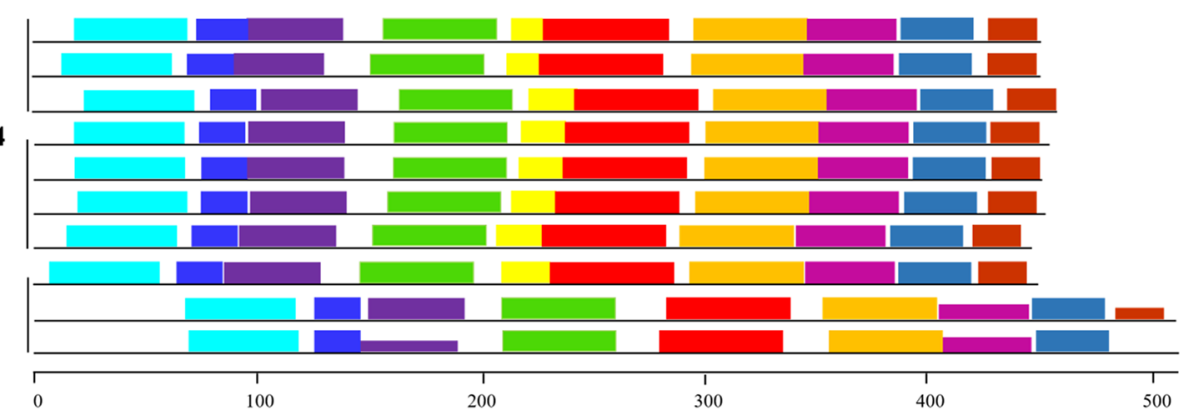

GDVAFAYAGHNVVLEIQATIPSTPEKPSKKPMWKGVVVAYIVVALCYFPV

[ REKAIDDWLPITSSRNAKWWYSAFHNVTAMVGAGVLSLPYAMSELGWGPG

KRFDRYHELGQHAFGEKLGLWIVVPQQLIVEVGVBIVYMVTG

DDNILITLSKPKWLIALANMMVVVHVIGSYQIYAMPVFDMJETVLVKKLR

DTTYFLPCIMWLAIYKPKRFSLSWFTNWGCIILG
KTTYFIMIFASVHFVLSQLPNFNSISGVSLAAAVMSLSYSTIAWGASVDK

- FPPGLTLRLIARSLYVAFTMFIAITFPFFGGLLGFFGGFAF

SWVITLYTLWQMVEMHEMVPG

DPIGGLRQIIDAKTYKFYS

VDYSLRATTTPGKVFGFFGAL

Fig. 5 (See legend on next page.) 
(See figure on previous page.)

Fig. 5 Phylogenetic relationship and protein motifs of OsLHT1 and its homologs. a Phylogenetic tree of OsLHT1 and its homologs from maize, sorghum, Brachypodium and Arabidopsis. With the exception of OsLHT1-6 and AtLHT1-10, the first two letters of each protein label represent the abbreviated species name, followed by GenBank accession number. Zm, Zea mays; Sb, Sorghum bicolor; Bd, Brachypodium distachyon. The phylogenetic tree was constructed by Mega 6.0 software using ClustalW for the alignment and the neighbour-joining method for the construction of the phylogeny [28]. The bootstrap values, shown at the nodes, are percentages for 1000 replications. The red triangle marks the OsLHT1. b Schematic representation of conserved motifs in OsLHT1-6 and its closest homologs in maize, sorghum, Arabidopsis and Brachypodium. Each motif is represented by different color boxes. The order of the motifs corresponds to their position within individual protein sequences

acids, but not basic amino acids. There are 6 LHT members existing in rice [14], but their substrate specificity has not yet been determined until now. OsLHT1 has been reported as a His transporter previously [22], and its substrate specificity was analyzed in Xenopus oocytes in the present study. We found that OsLHT1 could mediate the transport of various amino acids with different characteristics (Fig. 1a, b). Different from AtLHT1 expressed in yeast [18], the Lys transport activity of OsLHT1 was very weak relative to His (Fig. 1a, b). Moreover, the neutral amino acid Asn, but not the basic amino acid His, was the best substrate for OsLHT1 among the amino acids tested (Fig. 1a, b), so the substrate profile of OsLHT1 resemble that of AtLHT2 and AtLHT6 [20, 21]. However, it should be noted that OsLHT1 and AtLHT2 differ in substrate specificity and affinity. The $K_{\mathrm{m}}$ value of OsLHT1 for its best substrate Asn is $583 \pm 51 \mu \mathrm{M}$ (Fig. 1c), which is one order of magnitude higher than the values of AtLHT2 for Pro and Asp ( $K_{\mathrm{m}}=13,72 \mu \mathrm{M}$, respectively) [20]. The difference in $K_{\mathrm{m}}$ values between OsLHT1 and AtLHT2 may imply their different roles in planta, which will require further analysis in future study. Overall, given the substrate specificity of the LHTs identified so far, it could be concluded that the LHTs were not Lys and His selective transporters, but ones with broad substrate specificity and with preference for neutral and acidic amino acids.

The substrate profile of OsLHT1 was determined in Xenopus oocytes under pH5.4 condition. At pH 5.4, basic amino acids such as His are positively charged, while acidic amino acids such as Asp and Glu carry mainly negative net charges. However, all these amino acids with opposite charges could be transported by OsLHT1 (Fig. 1a, b). On the other hand, Lys and Arg carrying like charge as His were not efficiently transported by OsLHT1 (Fig. 1a, b). So it is supposed that net charge is not a major determinant of substrate recognition for OsLHT1. Besides charge, individual amino acids differ with respect to geometry, which might play a crucial role in substrate recognition by OsLHT1.

OsLHT1 was localized at the plasma membrane in rice protoplasts (Fig. 4b), which is consistent with its function as an amino acid transporter, and also agrees with previous work by Liu et al. [22] and Whiteman et al. [30]. In Liu's study, the OsLHT1-GFP fusion construct was expressed transiently in onion epidermal cells, and green fluorescence was detected almost exclusively at the plasma membrane [22]. Whiteman et al. [30] isolated rice plasma membrane and tonoplast proteins, and found that OsLHT1 specific peptides were present in plasma membrane fractions but not in tonoplast.

OsLHT1 was expressed in roots (Fig. 4a, Additional file: Figure S4), and could transport amino acids both in yeast and Xenopus oocytes (Fig. 1a) [22], which implied that it might be capable of mediating amino acids acquisition from soil. However, given the facts that: (1) free amino acid concentrations found in the bulk soil solution vary greatly, and rarely exceed $50 \mu \mathrm{M}$ [31-34]; (2) the $K_{\mathrm{m}}$ value of OsLHT1 for the most active substrate, Asn, was $583 \pm 51 \mu \mathrm{M}$ (Fig. 1c), which is much higher than the free amino acid concentrations in most soil solution; (3) a large number of microorganisms exist in soil, which are the superior competitors for free amino acids in comparison to plant roots, especially at low soil amino acids concentrations [35-37], we reasoned that the main function of OsLHT1 in planta might not be to acquire amino acids from soil, which needs to be verified in future studies. On the other hand, the absorbed inorganic $\mathrm{N}$ was converted into amino acids in roots or mature leaves (source), which are the major transport forms of $\mathrm{N}$ within plants, and were transported to various metabolically active organs (sink) such as root tips, young leaves, flowers and seeds. The source-to-sink translocation of amino acids is involved in several processes such as phloem loading, import into sinks and xylem-to-phloem transfer, which require the mediation of multiple amino acid transporters [11]. The amino acid permeases (AAPs) have been reported to be involved in the source-to-sink transport of amino acids [38-40]. For example, AtAAP8 is expressed in the source leaf phloem, and loss-of-function of AtAAP8 reduced a broad spectrum of amino acid phloem loading and partitioning to sinks, which in turn led to decreased silique and seed numbers in ataap 8 plants [40]. OsLHT1 was expressed in various shoot organs such as leaf, leaf blade, and localized to the plasma membrane (Fig. 4a, b), implying its function in amino acids translocation in planta. Furthermore, a lack of OsLHT1 function significantly inhibited plants development and reduced seed yields (Figs. 2e, 3a), so we supposed that OsLHT1 functions mainly in amino acids 
transport within plants, but the process it mediated will need further investigations in future.

\section{Conclusions}

Amino acid transporters mediate root amino acids uptake from soil, and their translocation within plants. Research on plant amino acid transporters had a strong focus on Arabidopsis, while our understanding of them is generally scant in rice. OsLHT1 was found to serve as a histidine transporter in yeast, but its substrate profile and function in planta are unclear. In the present study, we analyzed the substrate selectivity of OsLHT1 in Xenopus oocytes, and found that OsLHT1 could transport a broad spectrum of amino acids with preference for neutral and acidic amino acids. Spatial expression and subcellular analysis showed that OsLHT1 was expressed in various rice organs, and was targeted to the plasma membrane. A loss-of-function of OsLHT1 inhibited rice root and shoot growth, and markedly reduced grain yields. This study will further our understanding of the substrate specificity of OsLHT1 and its roles in rice growth promotion and yield formation.

\section{Methods}

\section{Plant materials}

The wild-type rice(cv. Nipponbare) and two oslht1 mutants were used in this study. The wild-type rice was ordered from rice resources conservation center of Guangxi University. The oslht1 mutants were constructed using the CRISPR-Cas9 gene-editing technology in our lab (see below), and its cultivation complies with China's legislation on genetically modified plants.

\section{Generation of OsLHT1 knockout lines}

The web-based tool CRISPR-P 2.0 software (http:// crispr.hzau.edu.cn/CRISPR2/) was used to design target sites of OSLHT1. Two target sequences were $5^{\prime}$ TAACTACCCACCGGCCAAGG-3' and 5'-TTCCCA TCACGTCGTCCAGG-3', respectively. The pCRISPROsLHT1 plasmid was constructed as described by $\mathrm{Ma}$ et al. [41]. Briefly, two sgRNA expression cassettes containing target sequence were constructed by overlapping PCR, and were cloned into pYLCRISPR/ Cas9Pubi with $B s a$ I restriction enzyme, resulting in the pCRISPR-OsLHT1.The resulting constructs were introduced into Nipponbare via Agrobacterium tumefaciens-mediated transformation. The $\mathrm{T} 0$ and $\mathrm{T} 1$ homozygous mutants were screened by PCR using primer pairs flanking two OsLHT1-specific target sites. Two independent knockout lines of OsLHT1 were selected for further phenotypic analysis.

\section{Morphological characterization}

Seedlings of wild-type and oslht1 plants were exposed to $0.5 \mathrm{mM} \mathrm{CaCl}_{2}$ solution at $28^{\circ} \mathrm{C}$ for $5 \mathrm{~d}$ (12-h-light and 12-h-dark condition), root length was measured by a ruler at different days. Field tests of rice were carried out at the experimental paddy field of Guangxi University in 2018, germinated seeds of wild type and mutants were cultured in the field. After three-leaf period, plants were transplanted to experiment field in March and harvested at the end of July. Agronomic traits including the plant height, stem length, tiller number, the grain yield per plant, panicle number per plant and grain number per panicle were recorded. The brown rice was weighed, and the 1000-grain weight and grain yield per plant were calculated.

\section{RNA isolation and gene expression analysis}

To investigate the expression pattern of OsLHT1, total RNA was extracted from different organs of 14-week-old WT rice using Trizol regent (Invitrogen). Reverse transcription was performed using $1 \mu \mathrm{g}$ of total RNA and the first-strand cDNA was reverse transcribed using superscript reverse transcriptase (Invitrogen) according to the manufacturer's instructions. The primers $5^{\prime}$-TCTT CGGTGGATTCGCCTTC-3' and 5'-ATGATGCAGAT CCAGTTGGTG-3' for OsLHT1, 5'-GGTCAACTTGT TGATTCCCCTCT-3' and 5'-AACCGCAAAATCCAA AGAACG-3' for Histone were used for qRT-PCR, and conditions for quantitative analysis were as follows: $94{ }^{\circ} \mathrm{C}$ for $2 \mathrm{~min}$, 35 cycles of $94{ }^{\circ} \mathrm{C}$ for $15 \mathrm{~s}, 60^{\circ} \mathrm{C}$ for $20 \mathrm{~s}$ and $72{ }^{\circ} \mathrm{C}$ for 30 s and final extension at $72^{\circ} \mathrm{Cfor} 10 \mathrm{~min}$. The gene transcript levels in each sample were normalized to that of the housekeeping gene Histone. Each experiment was conducted with three biological replicates.

\section{Subcellular localization of OsLHT1}

To investigate the subcellular location of OsLHT1, the full-length coding sequence of OsLHT1 (LOC_ Os08g03350) was amplified with primer pairs: $5^{\prime}$-CCCA AGCTTCGATGGGGACTCAGGTGGCAGATAA-3' and 5'-CGCGGATCCCTACGAGTAGAACTTGTATGTCT-

3', and was cloned into pYL322-GFP vector with HindIII and $B a m H$ I restriction sites for producing GFP-OsLHT1 construct. The plasmid GFP-OsLHT1 or GFP alone (control), along with mCherry-OsRac3 used as a plasma membrane marker [26], were cotransformed into the rice protoplasts by PEG-mediated transformation as previously described [26]. After transformation for 12 to $16 \mathrm{~h}$, the fluorescence signal images were observed via a confocal laser scanning microscope (Tcs sp8; Leica).

\section{Conserved motif and phylogenetic analysis}

Using the OsLHT1 sequence as the query, its homologs in maize, sorghum and Brachypodium distachyon were 
obtained from NCBI database by BLAST program. Multiple sequence alignments of OsLHT1 and its homologs were generated using ClustalW version1.83 with default settings, and the neighbor-joining (NJ) tree was constructed using neighbor-joining (NJ) method of MEGA 6.0 with 1000 bootstrap replications. To better understand the conserved structural features of LHT family proteins, the online tool MEME (Multiple Expectation Maximization for Motif Elicitation) was used to identify the conserved motifs in the encoded LHT family and each motif width was constrained between 3 and 50 residues.

\section{Electrophysiology}

The full-length coding sequence of OsLHT1 (LOC_ Os08g03350) was amplified by high-fidelity PCR from Nipponbare cDNA using gene specific primers 5'CGCTCAACTTTGGCAGATCTATGGGGACTCAGGT GGCAGATAAC-3' (initiation codon underlined) and 5'-AGATCCTAGTCAGTCACTAGTCTACGAGTAGA ACTTGTATGTC-3' (termination codon underlined). The resultant PCR product and the BglII/ Spe I linearized vector pT7Ts were recombined with a seamless assembly cloning kit (Vazyme) following the manufacturer's instructions. The ligated products were then introduced into $E$. coli $\mathrm{DH} 5 \alpha$ and verified by sequencing. The recombinant plasmid DNA was linearized with $X b a \mathrm{I}$, and the cRNA was transcribed in vitro using the mMessage mMachine T7 kit (Ambion). Xenopus oocytes were prepared as described previously [42]. Oocytes were injected with 23.0 ng of OsLHT1 cRNA or an equivalent volume of nuclease-free water, using Nanoliter 2000 microinjector, and then incubated for $2-4$ days at $19^{\circ} \mathrm{C}$ in ND96 solution containing $96 \mathrm{~mm} \mathrm{NaCl}, 2 \mathrm{mM} \mathrm{KCl}, 1.8 \mathrm{mM} \mathrm{CaCl}_{2}, 1 \mathrm{mM}$ $\mathrm{MgCl}_{2}, 5 \mathrm{mM}$ HEPES (pH 7.4) and $50 \mathrm{mg} / \mathrm{L}$ gentamicin. Whole-cell currents were recorded using the TEVC technique (AxoClamp 900A, Molecular devices), and data acquisition, data analysis were carried out using software Clampex, clampfit 10.3 (Molecular devices) and SigmaPlot 12.0 (Systat Software). For current recording as in Fig. 1a and b, oocytes were voltage-clamped at $-70 \mathrm{mV}$. For current recording as in Fig. 1c, the holding potential was $-40 \mathrm{mV}$, and Asn-induced currents were measured at membrane potentials from -140 to $+20 \mathrm{mV}$ in $20 \mathrm{mV}$ increments for $500 \mathrm{~ms}$. The basal superfusing solution contained: $90 \mathrm{mM} \mathrm{NaCl}, 2 \mathrm{mM} \mathrm{CaCl}, 2 \mathrm{mM} \mathrm{MgCl}$, and 4 mM MES, and amino acids were added at desired concentrations. The $\mathrm{pH}$ of all solutions was adjusted to 5.4 using Tris/MES.

\section{Statistical analysis}

All treatments were repeated at least two times, and the statistical analysis difference was indicated by asterisks. Significant levels was defined " $P<0.05$, " $P<0.01,{ }^{* * * *} P<0.001$.

\section{Additional file}

Additional file 1: Figure S1. Transcript level of OsLHT1 in the wild-type and os/ht1 plants. Figure S2. Germination rates of the wild-type and os/ht1 seeds. Figure S3. Grains of the wild-type and os/ht1 mutants. Figure S4. Transcript level of OsLHT1 in the developing organs. Table S1. Raw qRT-PCR data in this study (Fig. 4a). (DOCX 586 kb)

\section{Abbreviations}

BLAST: Basic Local Alignment Search Tool; GFP: Green Fluorescent Protein; LHT: Lysine and Histidine Transporter; PCR: Polymerase Chain Reaction; TEVC: Two-Electrode Voltage Clamp

\section{Acknowledgements}

Not applicable.

\section{Authors' contributions}

$J X$ conceived and designed the experiment. XW performed most of the experiments. GY, MS, HD, QW, ZW, SF, and YS participated in the research. GY drafted the manuscript. GY and JX revised the manuscript. All of the authors discussed the results and commented on the manuscript. All authors read and approved the final manuscript.

\section{Funding}

The research was funded by National Natural Science Foundation of China (31760065), Guangxi Natural Science Foundation (2016GXNSFFA380013), Innovation Project of Guangxi Graduate Education (YCSW2018044), HundredTalent Program of Guangxi (2014) and the Project of High Level Innovation Team and Outstanding Scholar in Guangxi Colleges and Universities (2016). The funders did not play any roles in the design of the study, collection, analysis and interpretation of the relevant data, and writing the manuscript.

\section{Availability of data and materials}

All data and materials generated or analyzed during this study are included in this article or are available from the corresponding author on reasonable request.

\section{Ethics approval and consent to participate}

Not applicable.

\section{Consent for publication}

Not applicable.

\section{Competing interests}

The authors declare that they have no competing interests.

\section{Author details}

${ }^{1}$ State Key Laboratory of Conservation and Utilization of Subtropical Agro-bioresources, College of Life Science and Technology, Guangxi University, Nanning 530005, China. ${ }^{2}$ State Key Laboratory of Soil and Sustainable Agriculture, Institute of Soil Science, Chinese Academy of Sciences, No. 71, East Beijing Road, Nanjing 210008, China.

Received: 13 December 2018 Accepted: 13 June 2019

Published online: 20 June 2019

\section{References}

1. Näsholm T, Kielland K, Ganeteg U. Uptake of organic nitrogen by plants. New Phytol. 2009;182:31-48.

2. Wright DE. Amino acid uptake by plant roots. Arch Biochem Biophys. 1962; 97:174-80.

3. Li H, Hu B, Chu C. Nitrogen use efficiency in crops: lessons from Arabidopsis and rice. J Exp Bot. 2017;68:2477-88.

4. $\mathrm{Xu} \mathrm{GH}$, Fan XR, Miller AJ. Plant nitrogen assimilation and use efficiency. Annu Rev Plant Biol. 2012;63:153-82.

5. Li ZC, Bush DR. pH-dependent amino acid transport into plasma membrane vesicles isolated from sugar beet (Beta vulgaris $L$.) leaves: 2 . Evidence for multiple aliphatic, neutral amino acid symports. Plant Physiol. 1991;96: 1338-44. 
6. Weston K, Hall JL, Williams LE. Characterization of amino acid transport in Ricinus communis roots using isolated membrane vesicles. Planta. 1995;196: 166-73.

7. Wyse RE, Komor E. Mechanism of amino acid uptake by sugarcane suspension cells. Plant Physiol. 1984;76:865-70.

8. Frommer WB, Hummel S, Riesmeier JW. Expression cloning in yeast of a CDNA encoding a broad specificity amino acid permease from Arabidopsis thaliana. Proc Natl Acad Sci U S A. 1993;90:5944-8.

9. Hsu LC, Chiou TJ, Chen L, Bush DR. Cloning a plant amino acid transporter by functional complementation of a yeast amino acid transport mutant. Proc Natl Acad Sci U S A. 1993;90:7441-5.

10. Pratelli R, Pilot G. Regulation of amino acid metabolic enzymes and transporters in plants. J Exp Bot. 2014;65:5535-56.

11. Tegeder M, Rentsch D. Uptake and partitioning of amino acids and peptides. Mol Plant. 2010;3:997-1011.

12. Cheng L, Yuan H, Ren R, Zhao S, Han Y, Zhou Q, Ke D, Wang Y, Wang L. Genome-wide identification, classification and expression analysis of amino acid transporter gene family in Glycine max. Front Plant Sci. 2016;7:515.

13. Rentsch D, Schmidt S, Tegeder M. Transporters for uptake and allocation of organic nitrogen compounds in plants. FEBS Lett. 2007;581:2281-9.

14. Zhao H, Ma H, Yu L, Wang X, Zhao J. Genome-wide survey and expression analysis of amino acid transporter gene family in rice (Oryza sativa L). PLoS One. 2012;7:e49210.

15. Ortiz-Lopez A, Chang HC, Bush DR. Amino acid transporters in plants. Biochim Biophys Acta. 2000;1465:275-80.

16. Williams $L$, Miller A. Transporters responsible for the uptake and partitioning of nitrogenous solutes. Annu Rev Plant Physiol Plant Mol Biol. 2001;52:659-88.

17. Tegeder M. Transporters for amino acids in plant cells: some functions and many unknowns. Curr Opin Plant Biol. 2012;15:315-21.

18. Chen L, Bush DR. LHT1, a lysine and histidine-specific amino acid transporter in Arabidopsis. Plant Physiol. 1997;115:1127-34.

19. Hirner A, Ladwig F, Stransky H, Okumoto S, Keinath M, Harms A, Frommer WB, Koch W. Arabidopsis LHT1 is a high-affinity transporter for cellular amino acid uptake in both root epidermis and leaf mesophyll. Plant Cell. 2006;18:1931-46.

20. Lee $\mathrm{YH}$, Tegeder M. Selective expression of a novel high-affinity transport system for acidic and neutral amino acids in the tapetum cells of Arabidopsis flowers. Plant J. 2004:40:60-74.

21. Perchlik M, Foster J, Tegeder M. Different and overlapping functions of Arabidopsis LHT6 and AAP1 transporters in root amino acid uptake. J Exp Bot. 2014;65:5193-204

22. Liu D, Gong W, Bai Y, Luo JC, Zhu YX. OsHT, a rice gene encoding for a plasma-membrane localized histidine transporter. J Integr Plant Biol. 2005; 47:92-9.

23. Fischer WN, Loo DD, Ludewig U, Boorer KJ, Tegeder M, Rentsch D, Wright $\mathrm{EM}$, Frommer WB. Low and high affinity amino acid $\mathrm{H}^{+}$-cotransporters for cellular import of neutral and charged amino acids. Plant J. 2002;29:717-31.

24. Taylor MR, Reinders A, Ward JM. Transport function of rice amino acid permeases (AAPs). Plant Cell Physiol. 2015:56(7):1355-63.

25. Winter D, Vinegar B, Nahal H, Ammar R, Wilson GV, Provart NJ. An "electronic fluorescent pictograph" browser for exploring and analyzing large-scale biological data sets. PLoS One. 2007;2(8):e718.

26. Chen L, Shiotani K, Togashi T, Miki D, Aoyama M, Wong HL, Kawasaki T, Shimamoto K. Analysis of the Rac/Rop small GTPase family in rice: expression, subcellular localization and role in disease resistance. Plant Cell Physiol. 2010;51:585-95.

27. Tegeder M, Ward JM. Molecular evolution of plant AAP and LHT amino acid transporters. Front Plant Sci. 2012;3:21

28. Tamura K, Stecher G, Peterson D, Filipski A, Kumar S. MEGA6: molecular evolutionary genetics analysis version 6.0. Mol Biol Evol. 2013;30:2725-9.

29. Guether M, Volpe V, Balestrini R, Requena N, Wipf D, Bonfante P. LjLHT1.2-a mycorrhiza-inducible plant amino acid transporter from Lotus japonicus. Biol Fertil Soils. 2011:47:925-36.

30. Whiteman SA, Nühse TS, Ashford DA, Sanders D, Maathuis FJ. A proteomic and phosphoproteomic analysis of Oryza sativa plasma membrane and vacuolar membrane. Plant J. 2008:56:146-56.

31. Jämtgård S, Näsholm T, Huss-Danell K. Nitrogen compounds in soil solutions of agricultural land. Soil Biol Biochem. 2010;42:2325-30.

32. Jones DL, Owen AG, Farrar JF. Simple method to enable the high resolution determination of total free amino acids in soil solutions and soil extracts. Soil Biol Biochem. 2002;34:1893-902.
33. Monreal CM, McGill WB. Centrifugal extraction and determination of free amino acids in soil solutions by TLC using tritiated 1-fluoro-2,4dinitrobenzene. Soil Biol Biochem. 1985;17:533-9.

34. Svennerstam H, Jämtgård S, Ahmad I, Huss-Danell K, Näsholm T, Ganeteg U. Transporters in Arabidopsis roots mediating uptake of amino acids at naturally occurring concentrations. New Phytol. 2011;191:459-67.

35. Jones DL, Shannon D, Junvee-Fortune T, Farrar JF. Plant capture of free amino acids is maximized under high soil amino acid concentrations. Soil Biol Biochem. 2005;37:179-81.

36. Owen AG, Jones DL. Competition for amino acids between wheat roots and rhizosphere microorganisms and the role of amino acids in plant $\mathrm{N}$ acquisition. Soil Biol Biochem. 2000;33:651-7.

37. Vinolas LC, Healey JR, Jones DL. Kinetics of soil microbial uptake of free amino acids. Biol Fertil Soils. 2001;33:67-74.

38. Hunt E, Gattolin S, Newbury HJ, Bale JS, Tseng HM, Barrett DA, Pritchard J. A mutation in amino acid permease AAP6 reduces the amino acid content of the Arabidopsis sieve elements but leaves aphid herbivores unaffected. J Exp Bot. 2010;61:55-64.

39. Sanders A, Collier R, Trethewy A, Gould G, Sieker R, Tegeder M. AAP1 regulates import of amino acids into developing Arabidopsis embryos. Plant J. 2009:59:540-52.

40. Santiago JP, Tegeder M. Connecting source with sink: the role of Arabidopsis AAP8 in phloem loading of amino acids. Plant Physiol. 2016; 171:508-52.

41. Ma X, Zhang Q, Zhu Q, et al. A robust CRISPR/Cas9 system for convenient, high-efficiency multiplex genome editing in monocot and dicot plants. Mol Plant. 2015:8:1274-84

42. Schmidt A, Su YH, Kunze R, Warner S, Hewitt M, Slocum RD, Ludewig U, Frommer WB, Desimone M. UPS1 and UPS2 from Arabidopsis mediate high affinity transport of uracil and 5-fluorouracil. J Biol Chem. 2004;279:44817-24.

\section{Publisher's Note}

Springer Nature remains neutral with regard to jurisdictional claims in published maps and institutional affiliations.

Ready to submit your research? Choose BMC and benefit from:

- fast, convenient online submission

- thorough peer review by experienced researchers in your field

- rapid publication on acceptance

- support for research data, including large and complex data types

- gold Open Access which fosters wider collaboration and increased citations

- maximum visibility for your research: over $100 \mathrm{M}$ website views per year

At $\mathrm{BMC}$, research is always in progress.

Learn more biomedcentral.com/submissions 